The "effective reproduction rate" $(\mathrm{R})$ is the average number of new infections that each case generates. If $\mathrm{R}$ is 1 then a state of equilibrium exists. If $\mathrm{R}$ is less than 1 then the disease in question will eventually become extinct. For measles $\mathrm{R}$ is about 16 . This means that each case of measles can expect to generate about 16 new cases in a susceptible population. The aim of herd immunity is to reduce $\mathrm{R}$ to less than 1 for each disease thus stopping the disease from propagating in the community.

Over time, as the proportion of children who are immunised in a population increases, the number of new cases of a disease should drop. If, however, enough parents decide not to have their children vaccinated, more cases will start to appear and then the entire population is put at risk. Successful herd immunity relies on health workers and parents' cooperation to immunise sufficient numbers of children.

Despite recent further evidence of vaccine safety, health scares about the measles, mumps, and rubella vaccine have contributed to a $5 \%$ reduction in the number of children being immunised with this vaccine in the United Kingdom. This reduction is sufficient to allow the reproduction rate to start rising again, and as a result a new measles epidemic has been predicted in the United Kingdom within the next two years. ${ }^{2}$

Abi Berger science editor, BMJ

1 Begg NT, Gay NJ. Theory of infectious disease transmission and herd immunity. In: Balows A, Sussman M, eds. Topley and Wilson's microbiology and microbial infections. Vol 3. 9th ed. London: Edward Arnold, 1997.

2 Fall in MMR vaccine coverage reported as further evidence of vaccine safety is published. CDR Weekly 1999;9:227-30.

\title{
The SCOFF questionnaire: assessment of a new screening tool for eating disorders
}

\author{
John F Morgan, Fiona Reid, J Hubert Lacey
}

Eating disorders are among the most common psychiatric disorders in young women. Early detection and treatment improves prognosis, but presentation is often cryptic-for example, via physical symptoms in primary care. Ability to diagnose the condition varies and can be inadequate, ${ }^{1}$ and existing questionnaires for detection $^{23}$ are lengthy and may require specialist interpretation. No simple, memorable screening instruments are available for non-specialists. In alcohol misuse the CAGE questionnaire ${ }^{4}$ has proved popular with clinicians because of its simplicity. We developed and tested a similar tool for eating disorderswith questions designed to raise suspicion that an eating disorder might exist-before rigorous clinical assessment.

\section{Participants, methods, and results}

We developed five questions addressing core features of anorexia nervosa and bulimia nervosa using focus groups of patients with eating disorders and specialists in eating disorders; we tested the questions in a feasibility study of patients and staff at an eating disorders unit. None of these participants was involved in the subsequent study. We created the acronym SCOFF from the questions (box).

We recruited cases sequentially from referrals to a specialist clinic: 116 women aged 18-40 years who were confirmed as having either anorexia nervosa $(n=68)$ or bulimia $(n=48)$, according to the criteria specified in the Diagnostic and Statistical Manual of Mental Disorders, fourth edition. We recruited 96 women aged 18-39 as controls; these women, recruited through advertising by local colleges, were confirmed as not having an eating disorder. Cases and controls were asked the SCOFF questions orally; they also completed the eating disorder inventory ${ }^{3}$ and the BITE self rating scale for bulimia. ${ }^{2}$

\section{The SCOFF questions* \\ Do you make yourself Sick because you feel uncomfortably full? \\ Do you worry you have lost Control over how much you eat? \\ Have you recently lost more than One stone in a 3 month period? \\ Do you believe yourself to be Fat when others say you are too thin? \\ Would you say that Food dominates your life? \\ *One point for every "yes"; a score of $\geqslant 2$ indicates a likely case of anorexia nervosa or bulimia}

No significant differences existed between cases and controls for age or ethnicity. As expected, more cases than controls were in the highest socioeconomic groups $\left(\left(\mathrm{P}<0.001, \chi^{2}=47.4, \mathrm{df}=3\right)\right.$, and cases were more likely to be single, separated, or divorced $\left(\mathrm{P}<0.001, \chi^{2}=13.0, \mathrm{df}=1\right)$. Mean length of illness for cases was 8 years (SD 4.81; range 1-25). Mean body mass index (weight $(\mathrm{kg}) /\left(\right.$ height $\left.(\mathrm{m})^{2}\right)$ ) for controls, bulimic cases, and anorectic cases was 22.3 (SD 1.90), 24.4 (1.77), and 15.1 (0.76) respectively. All scores on the eating disorder inventory and the BITE scale were consistent with published data for women with or without eating disorders. ${ }^{2}{ }^{3}$

All participants found the questions and the term SCOFF acceptable. Setting the threshold at two or more positive answers to all five questions provided $100 \%$ sensitivity for anorexia and bulimia, separately and combined (all cases, 95\% confidence interval $96.9 \%$ to $100 \%$; bulimic cases, $92.6 \%$ to $100 \%$; anorectic cases, $94.7 \%$ to $100 \%$ ), with specificity of $87.5 \%$ (79.2\% to $93.4 \%$ ) for controls (table).

\section{Department of Psychiatry, St George's Hospital Medical School, London SW17 ORE \\ John F Morgan clinical research fellow $\mathrm{J}$ Hubert Lacey professor \\ Department of Public Health Sciences, \\ St George's \\ Hospital Medical \\ School \\ Fiona Reid \\ lecturer in medical statistics \\ Correspondence to: J F Morgan jmorgan@sghms. ac.uk}

BMJ 1999;319:1467-8 
Numbers of cases (true positives) and controls (true negatives) identified by SCOFF questionnaire* as being likely to have eating disorder

\begin{tabular}{lcc} 
& $\begin{array}{c}\text { Total No of } \\
\text { subjects }\end{array}$ & $\begin{array}{c}\text { No of participants identified } \\
\text { by SCOFF as likely to have } \\
\text { eating disorder }\end{array}$ \\
\hline Cases & & \\
\hline All cases & 116 & 116 \\
\hline Bulimic cases & 48 & 48 \\
\hline Anorectic cases: & 68 & 68 \\
\hline "Bingeing" & 35 & 35 \\
\hline "Restricting" & 33 & 33 \\
\hline Controls & 96 & 12 \\
\hline
\end{tabular}

*If participants gave positive responses to at least two of the five questions (see box).

\section{Comment}

The SCOFF questionnaire seems highly effective as a screening instrument for detecting eating disorders; it is simple, memorable, and easy to apply and score, and it is designed to raise suspicion of a likely case rather than to diagnose.

We consider that the SCOFF questionnaire performed well against the 10 questions suggested by Greenhalgh to assess screening tests. ${ }^{5}$ The false positive rate of $12.5 \%$ is an acceptable trade off for very high sensitivity.
Further work is needed to establish validity and reliability in a wider population, and particularly in those at risk of eating disorders in the general population. None the less, there is sufficient evidence of validity for it to be used routinely in all patients considered at risk of eating disorders.

We thank Professor Martin Bland, professor in medical statistics, for guidance in planning the research methodology.

Contributors: JFM initiated and coordinated the study, discussed core ideas, designed the study protocol, and participated in data collection and analysis and in the writing of the paper. JHL discussed core ideas, participated in protocol design and interpretation of data, and contributed to the writing of the paper. FR led the statistical analysis, participated in data interpretation, and contributed to the writing of the paper. JFM will act as guarantor of the paper.

Funding: None.

Competing interests: None declared.

1 King MB. Eating disorders in a general practice population. Prevalence, characteristics and follow-up at 12 to 18 months. Psychol Med Monogr Suppl 1989;16:191-4.

2 Henderson M, Freeman CPL. A self-rating scale for bulimia. The "BITE" Br J Psychiatry 1987;150:18-24.

3 Garner DM, Olmstead MA, Polivy J. Development and validation of a multidimensional eating disorder inventory for anorexia nervosa and bulimia. Int J Eating Disorders 1983;2:15-34.

4 Ewing JA. Detecting alcoholism-the Cage questionnaire. JAMA 1984;252:1905-7.

5 Greenhalgh T. Papers that report diagnostic or screening tests. BMJ 1997;315:540-3

(Accepted 16 August 1999)

\section{A memorable period A small United Nations}

In King's Cross in London in 1856 a small independent, voluntary hospital assembled in a modest house, 11 York Way. Sherard Freeman Statham, assistant surgeon at University College, founded the institution and named it the Great Northern Hospital. On 17 July 1888 the hospital moved to a new building in the Holloway Road and was formally opened by the Prince and Princess of Wales. It received a royal charter on 2 November 1921. On its first centenary the hospital had 279 beds and a bustling outpatient department. The later half of the present century saw the Royal Northern Hospital transform into a major centre of clinical excellence and postgraduate education. The reputation was based on the century old tradition started by medical luminaries such as Dr Robert Bridges, who later became poet laureate; Sir Andrew Clark, president of the Royal College of Physicians; and Sir William Savory, president of the Royal College of Surgeons. These outstanding personalities were to be followed by surgical teachers, including Sir Lancelot Barrington-Ward, Mr Hamilton Bailey, and Mr McNeil Love, and physicians, such as Dr Clifford Beale and Dr Tommy Horder (later Lord Horder). My association with the hospital started in October 1966. A Prophit Research Scholarship from the Royal College of Physicians made it possible for me to stay there for three years and carry out clinical and immunological studies in sarcoidosis. In 1953 Dr David Geraint James spearheaded the multidisciplinary sarcoidosis clinic, the first clinic of its kind. It was unique because he involved clinicians, physiologists, and immunologists. Scattered among them were residents, postgraduate and medical students, and international visitors. Our patients came from all walks of life. The sarcoidosis clinic became and remained for more than three decades a small United Nations. Weekly staff conferences were dominated by the sharp wit of Sir Reginald Murley, later president of the Royal College of Surgeons, and his team, and the postgraduate dean, Dr Geraint James, and his team. These gatherings were occasions of sophisticated, intellectual debate, lightened by agreeable repartee. I recall these weekly meetings as the most lively and rewarding in Britain. During my three years I was fortunate to meet and exchange ideas with Nobel laureates Peter Medawar and Baruch Blumberg; geneticist Alexander Bearn; hepatologist Sheila Sherlock; gastroenterologist Christoper Booth; surgeon Dickson Wright; endocrinologists Raymond Greene and Sir John McMichael; sarcoidologists Guy Scadding, Sven Lofgren, and Louis Siltzbach; and immunologist Jan Costa Waldenstrom. The Royal Northern Hospital was closed as a result of a game of political chess. There were three other important hospitals in north London - the Royal Free, University College, and the Whittington. A process of amalgamation united all three, and the Royal Northern lost out in the reshuffle. The site is now a large and expensive block of flats. I hope the present residents enjoy themselves as much as we did.

Om P Sharma, professor of medicine, Los Angeles

We welcome articles of up to 600 words on topics such as A memorable patient, A paper that changed my practice, My most unfortunate mistake, or any other piece conveying instruction, pathos, or humour. If possible the article should be supplied on a disk. Permission is needed from the patient or a relative if an identifiable patient is referred to. We also welcome contributions for "Endpieces," consisting of quotations of up to 80 words (but most are considerably shorter) from any source, ancient or modern, which have appealed to the reader. 